\title{
Photonic crystal fibers as miniature monitoring platforms for petroleum characterization
}

Rodolfo Luiz Patyk, Cicero Martelli, Marco J. da Silva, Rigoberto E. M. Morales, Masood Naqshbandi, et al.

Rodolfo Luiz Patyk, Cicero Martelli, Marco J. da Silva, Rigoberto E. M. Morales, Masood Naqshbandi, John Canning, "Photonic crystal fibers as miniature monitoring platforms for petroleum characterization," Proc. SPIE 8421, OFS2012 22nd International Conference on Optical Fiber Sensors, 84217H (7 November 2012); doi: 10.1117/12.975632

Event: OFS2012 22nd International Conference on Optical Fiber Sensor, 2012, Beijing, China 


\title{
Photonic Crystal Fibers as Miniature Monitoring Platforms for Petroleum Characterization
}

\author{
Rodolfo Luiz Patyk $^{\mathrm{a}}$, Cicero Martelli*a ${ }^{\mathrm{a}}$, Marco J. da Silva ${ }^{\mathrm{a}}$, Rigoberto E.M. Morales ${ }^{\mathrm{b}}$, Masood \\ Naqshbandi ${ }^{\mathrm{c}}$, John Canning ${ }^{\mathrm{c}}$ \\ ${ }^{a}$ Graduate School on Electrical Engineering and Applied Computer Science, and \\ ${ }^{\mathrm{b}}$ Department of Mechanical Engineering, Federal University of Technology - Paraná, Curitiba PR \\ Brazil, 80230-901 \\ 'Interdisciplinary Photonics Laboratory, School of Chemistry, The University of Sydney - Sydney \\ NSW 2006 Australia
}

\begin{abstract}
A fiber design that allows the characterization of high and low refractive index materials is proposed and demonstrated. This fiber consists of an air-silica photonic crystal fiber supporting a Gaussian like mode confined in the fiber core and a ring mode in a region between the structured area and the fiber cladding. This versatile fiber design finds applications in the oil industry where materials of different refractive indices are found. The characterization of petroleum and $\mathrm{CO}_{2}$ using the new fiber is demonstrated.
\end{abstract}

Keywords: Photonic crystal fiber, microstructured optical fiber, petroleum.

\section{INTRODUCTION}

Photonic crystal fibers (PCF) have long been used as sensing systems to characterize the optical properties of materials placed inside of their holes. Long interaction lengths, broad bandwidth, small sample volume, ease to control and stabilize the sample temperature are some of the main properties that make PCF fibers attractive for chemical sensing. Examples of absorption and refractive index monitoring using structured fibers are found in the literature and include liquid samples ${ }^{1}$, thin films deposited inside of the fiber holes ${ }^{2}$ and the phase change of liquids and solids ${ }^{3}$. One of the main limitations for all photonic crystal fiber designs is the refractive index of the samples. For the diffractive fibers with hollow cores the insertion of any liquid or solid material in the holes leads directly to the fiber transmission bandwidth change that can turn wavelength region of interest completely not guided. As for the solid core fibers the main problem concerns the materials with refractive indices higher than that of the fiber host material, which is conventionally made of silica with $\mathrm{n}_{\mathrm{SiO} 2}=1.45$ at $1550 \mathrm{~nm}$. Both fiber designs will also suffer from power reference shifting as new materials are inserted in the holes and the guidance properties of the fibers change. Here a fiber design that is able to operate with high and low refractive index materials inserted in the holes is proposed (Figure 1).
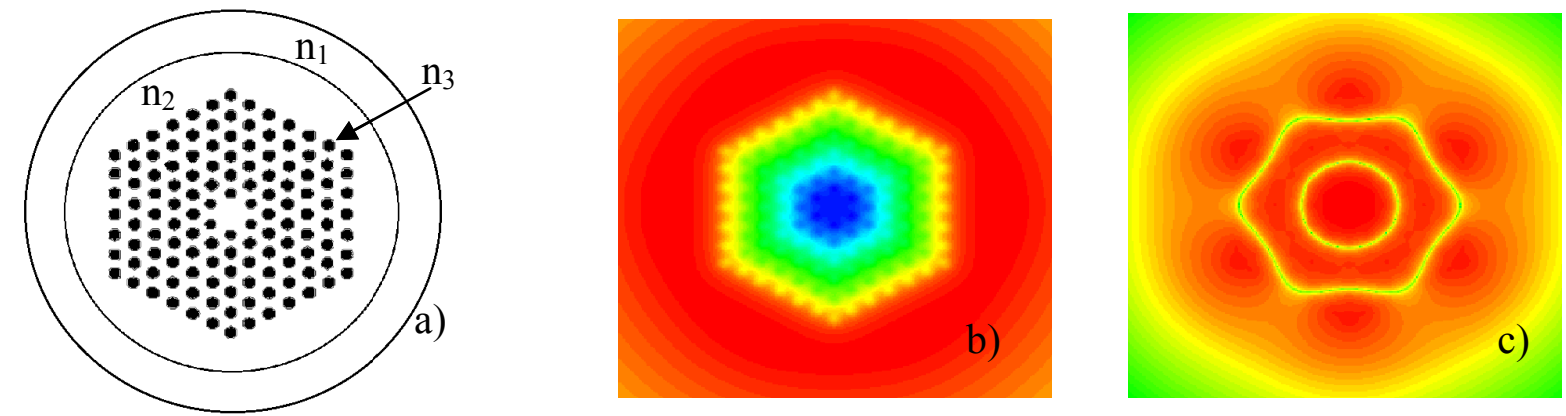

Figure 1. a) Cross-section profile of the proposed PCF fiber for petroleum monitoring; b) log scale of the numerical calculation of the near field profile for a ring like mode confined between the structured region and the outer most ring of silica in the absence of petroleum in holes, and; c) log scale of the numerical calculation of the near field profile for a ring like mode with the insertion of petroleum into the fiber holes. Mode plots are in the same scale.

*c.martelli@utfpr.edu.br; phone 55413310 4685; www.utfpr.edu.br 
The proposed PCF fiber is made of three materials as depicted in Figure 1.a: $\mathrm{n}_{1}$ and $\mathrm{n}_{2}$ which are silica of different density and purities and $n_{3}$ which is the material of the holes. The fiber has two main modes at the $1550 \mathrm{~nm}$ region, one confined in the core within the holey region and another with a ring like shape confined between the structured region and the interface of $\mathrm{n}_{2}$ and $\mathrm{n}_{1}$ materials. The fiber structure is made of 6 rings of holes distributed in a hexagonal lattice with $\Lambda \sim 1.41 \mu \mathrm{m}$ and $\mathrm{d} \sim 0.8 \mu \mathrm{m}$ and core diameter of approximately $2.4 \mu \mathrm{m}$.

The core mode is only supported for hole refractive indices smaller than silica's RI. Hence, this fiber is the ideal for gas monitoring that have the RI very close to 1.0 or liquids of low refractive index. The ring like mode is supported for both high and low refractive index materials within the fiber holes. Figure 1.b shows the energy distribution of the ring like mode cross section when there is only air in the holes. It is possible to observe that although most of the energy is being confined in the ring region outside the holey structure some of the power penetrates the holes interacting with the holes materials. When a high refractive index material is inserted into the fiber holes most of the energy of the ring mode is dragged into the holey region and peaks of higher energy are found on top of the holes. For the calculation presented in Figure 1.c the refractive index of the hole material was considered the same as a crude oil's, i.e. $n_{\text {hole }}=n_{\text {petroleum }}=1.5^{4}$. It is worth pointing out that no proper confinement was observed for the core mode for $n_{\text {hole }}>n_{\mathrm{SiO} 2}$ and that the calculated confinement losses for the ring mode in both case $\mathrm{n}_{\text {hole }}=\mathrm{n}_{\text {air }}$ and $\mathrm{n}_{\text {hole }}=\mathrm{n}_{\text {petroleum }}$ were negligible. Therefore, this fiber design seems to be ideal for the characterization of virtually any material that can be inserted into fiber holes. Given the importance of the petroleum industry as well as its constant need for novel solutions that can be integrated into the always limited space found in the production columns and in the subsea equipment this fiber is being used for the development of a miniaturized sensing system to characterize petroleum and all other substances that can be found in the reservoirs. Carbon dioxide is one of these substances which are abundantly found in the Brazilian offshore reserves and is consequently of interest for petroleum chemical sensing system. Results of the petroleum characterization as well as the detection of $\mathrm{CO}_{2}$ using the proposed PCF fiber are presented in the following sections.

\section{CARBON DIOXIDE DETECTION}

Most of the optical sensors found commercially or in the literature for $\mathrm{CO}_{2}$ detection operate at the mid-infrared wavelengths where the optical absorption of this molecule is highest. The down side of operating in such IR region is the lack of optical components such as sources and detectors as well as waveguides that can considerably increase the optical interaction lengths and subsequently the sensitivity of the sensor. Carbon dioxide presents a weak absorption band around $1570 \mathrm{~nm}$ which coincides with one important telecom band and its detection could therefore benefit from the components availability. The long interaction lengths possible by the PCF fibers appear as an alternative solution for the low absorption coefficient of $\mathrm{CO}_{2}$ at $1570 \mathrm{~nm}$ and allow the use of all components developed by telecommunication industry that can be found off the shelf at low prices. Figure 2.a show the absorption spectrum of carbon dioxide at the $1570 \mathrm{~nm}$ wavelength region measure by an ASE source and an optical spectrum analyzer along with the PCF described above. Approximately $70 \mathrm{~m}$ of the PCF fiber was filled with a mixture of $\mathrm{CO}_{2}$ and air and had its ends spliced to two smf 28 patch cords. During the splicing procedure care was taken so that only the ring like mode was excited demonstrating the sensing concept. Figure 2.b shows a reference absorption spectrum measured at a free space calibration chamber. The $\mathrm{CO}_{2}$ absorption at $1570 \mathrm{~nm}$ is a combination of the bands: $2 \mathrm{v}_{1}+2 \mathrm{v}_{2}+\mathrm{v}_{3}$.
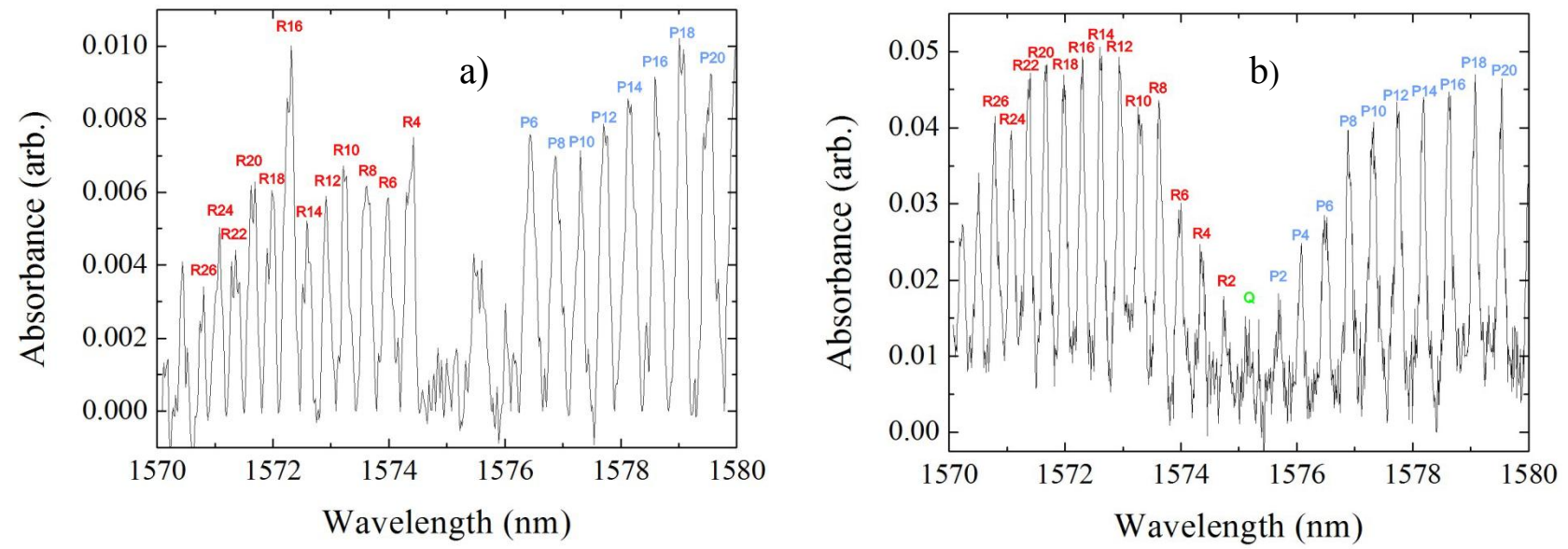

Figure 2. a) Optical absorption of $\mathrm{CO}_{2}$ at $1570 \mathrm{~nm}$ using a PCF fiber and b) using a reference chamber. 
It is possible to observe that the overall features of the absorption spectrum are preserved including the narrow bands and so on for the fiber measurement. The lack of nanometer positioning precision at the splicing for the PCF reference spectrum and $\mathrm{CO}_{2}$ spectrum can be accounted for the small distortions in the band intensity distributions.

\section{PETROLEUM PCF FIBER CHARACTERIZATION}

Crude oil was infiltrated inside of the PCF fiber holes using a pressure chamber. The total fiber length was $150 \mathrm{~mm}$ and after the fiber was completely filled with petroleum one end was spliced to a smf 28 patch cord. Before studying the optical properties of the PCF fiber infiltrated with petroleum an optical absorption spectrum of the crude oil was measured so that the wavelength region where absorption is lower could be investigated with the optical fiber. The measurement was carried out using a supercontinuum broad band optical source and two optical spectrum analyzers operating from $350 \mathrm{~nm}$ all the way up to $2400 \mathrm{~nm}$. An oil film of approximately $2 \mathrm{~mm}$ of thickness was deposited on a quartz slide and the light transmission of the film was measured. Figure 3.a shows the corresponding absorption spectrum. Light absorption is found over the entire spectrum with high absorption bands in the visible region and also centered at 900 and $2300 \mathrm{~nm}$. Lower absorption is present around 1500-1700 nm which coincidently is the same spectral region where $\mathrm{CO}_{2}$ has some absorption as described above. One could easily predict that with the right fiber design this spectral region can be used to detect $\mathrm{CO}_{2}$ mixed with petroleum, an important issue in the oil industry.

The PCF fiber filled with crude oil was characterized by measuring its transmission spectrum at $1550 \mathrm{~nm}$. The attenuation coefficient determined by a cut back test at the $1570 \mathrm{~nm}$ is showed in Figure 3.b. The optical attenuation coefficient for the petroleum filled PCF was measured to be approximately $0.2 \mathrm{dBm} / \mathrm{mm}$.
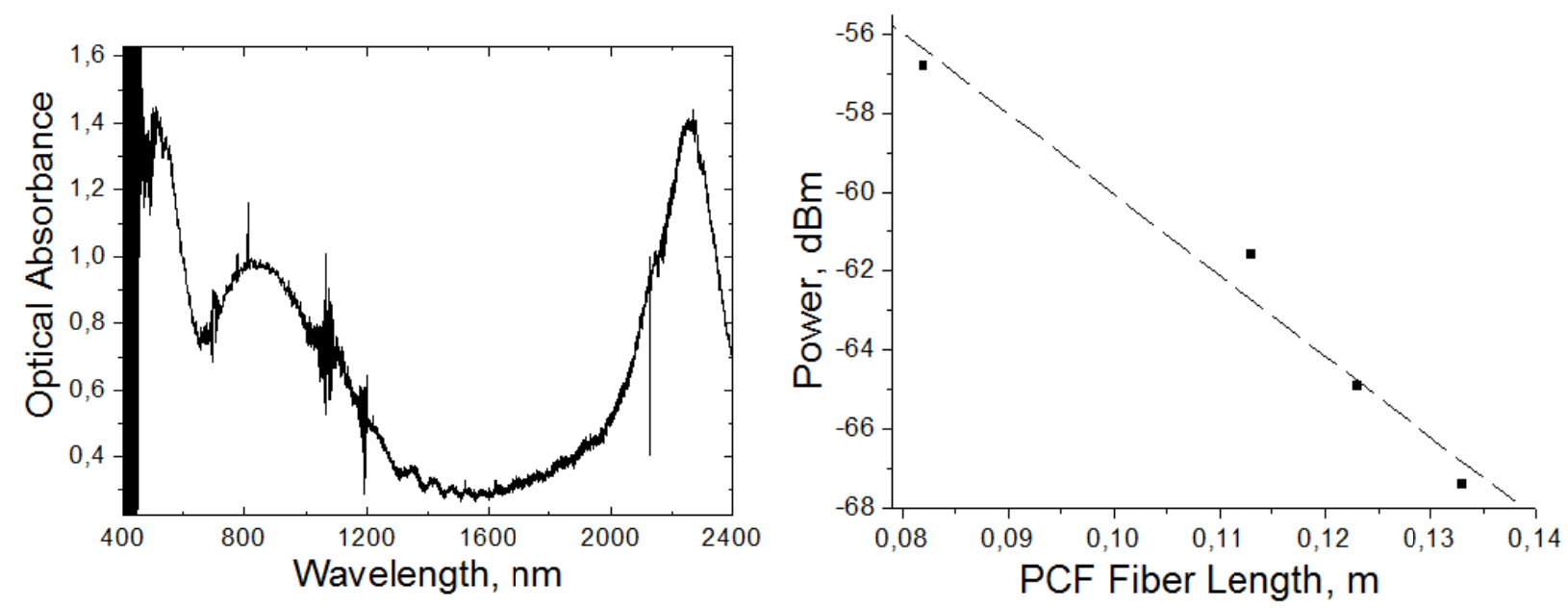

Figure 3. a) Optical absorption of a $2 \mathrm{~mm}$ thick petroleum film, and; b) cut back test for determining the petroleum filled PCF fiber absorption coefficient.

This high attenuation appears as a result of the optical mode field being coupled directly over the fiber holes as a result of refractive rise with the insertion of petroleum in the holes. This significantly increases the overlap of the mode field with the sample which in terms means that the system is much more sensitive and shorter lengths of fibers are needed. It is important to point out that this high overlap is only possible because of the refractive index contrast between the outermost ring with RI equals $\mathrm{n}_{1}$ which is higher the $\mathrm{n}_{2}$ the refractive index of the structure host material. Standard PCF fibers would not be able support such modes and hence would not reach this level of sensitivity. It is somewhat expectable that the optical properties of this PCF fiber, e.g. mode field overlap with the holey region, can be tailored by engineering the microstructure and the RI of the fiber materials.

\section{CONCLUSION}

A PCF fiber able to detect materials with refractive indices lower and higher than silica's RI was proposed and demonstrated and its application in the oil industry was discussed. Results for the detection of $\mathrm{CO}_{2}$ as well as crude oil inside of the fiber holes were presented and show that this consists of promising technique for petroleum 
characterization. More generically the possibility of spreading the mode field over the fiber holes shines new light in the opportunities for optical detection and characterization of chemical samples. Here extremely high sensitivity was achieved leading to a strong attenuation of the fiber transmission at the $1570 \mathrm{~nm}$. Furthermore, pure silica photonic crystal fibers are also important devices for harsh environments applications such as the oil industry given their temperature stability and chemical inertia especially with regards to hydrogen ${ }^{5}$.

\section{ACKNOWLEDGMENTS}

The authors acknowledge the financial support of CNPq, CAPES, FINEP and Petrobras, in Brazil, and ARC in Australia. This project is developed under the Petrobras`s R\&D program.

\section{REFERENCES}

[1] C. Martelli, J. Canning, D. Stocks, M. Crossley, "Water-soluble porphyrin detection in a pure-silica photonic crystal fiber", Opt. Lett., 31, 2100-2102, (2006)

[2] C. Martelli, J. Canning, J. R. Reimers, M. Sintic, D. Stocks, T. Khoury, M. J. Crossley, "Evanescent-Field Spectroscopy using Structured Optical Fibers: Detection of Charge-Transfer at the Porphyrin-Silica Interface", J. Am. Chem. Soc., 2009, 131 (8), 2925-2933 (2009)

[3] C. Martelli, J. Canning, M. Kristensen, N. Groothoff, "Refractive index measurement within a photonic crystal fibre based on short wavelength diffraction", Sensors, 7, 2492-2498 (2007)

[4] H. El Ghandoor, E. Hegazi, IbraheemNasser, G.M. Behery, "Measuring the refractive index of crude oil using a capillary tube interferometer", Optics \& Laser Technology 35, 361 - 367 (2003)

[5] C. Martelli A. L. C. Triques, A. M. B. Braga, K. Cook, J. Canning V. Takahashi, "Operation of Optical Fiber Sensors in Hydrogen-Rich Atmosphere" In: European Workshop on Optical Fiber Sensors, 2010, Porto - Portugal, 2010 